\title{
Outpatient transplantation in the COVID-19 era: a single-center Latin American experience
}

\author{
Perla R. Colunga-Pedraza ${ }^{1}$ - Julia E. Colunga-Pedraza ${ }^{1}$ Jesús D. Meléndez-Flores ${ }^{1} \cdot$ Mariana González-Treviño ${ }^{1}{ }^{1}$ • \\ Eugenia M. Ramos-Dávila $\mathbb{D}^{1}$ - Andrés Gómez-De León $\mathbb{1}^{1}$. Samantha P. Peña-Lozano $\mathbb{D}^{1}$ • Óscar González-Llano ${ }^{1}$. \\ Olga G. Cantú-Rodríguez $\mathbb{D}^{1} \cdot$ Mónica Bustillos-Muñoz ${ }^{1} \cdot$ César Homero Gutiérrez-Aguirre $\mathbb{D}^{1}$ • \\ David Gómez-Almaguer $\mathbb{D}^{1} \cdot$ José C. Jaime-Pérez $\mathbb{D}^{1}$
}

Received: 2 March 2021 / Revised: 6 May 2021 / Accepted: 17 May 2021 / Published online: 27 May 2021

(c) The Author(s), under exclusive licence to Springer Nature Limited 2021

\section{To the Editor:}

The COVID-19 pandemic has placed an unprecedented strain on healthcare systems worldwide, which has been particularly challenging in resource-constrained settings. Latin America has some of the highest COVID-19 death rates, highlighting its fragile healthcare systems. [1] The Latin-American Blood and Marrow Transplantation group (LABMT) reported a decrease in hematopoietic cell transplantations (HCT) during 2020, reflecting the impact of the pandemic. [2] There is controversy regarding whether HCT during the COVID-19 pandemic is feasible and safe, [3-6] since HCT is usually a myeloablative procedure requiring inpatient care, which increases the exposure of these immunocompromised patients to SARS-CoV-2 when a conventional HCT unit is not available. For twenty years our center has pioneered the outpatient HCT method, with comparable outcomes to centers in high-income countries. [7] We report outcomes of our ambulatory HCT program during the COVID-19 pandemic from March to December 2020.

To undergo outpatient HCT, patients were required to have a Karnofsky/Lansky score $\geq 70 \%$, serum creatinine $<2$ $\mathrm{mg} / \mathrm{dL}$, temporary residence near the hospital, and an adequate caregiver. The patient and family received clear information on the risks and signed informed consent.

Operational changes during the COVID-19 pandemic in our center are displayed in Table 1 .

José C. Jaime-Pérez

carjaime@hotmail.com

1 Hematology Service, Internal Medicine Division, Dr. Jose Eleuterio Gonzalez University Hospital and School of Medicine, Universidad Autónoma de Nuevo Leon, Monterrey, Mexico
Patients, donors, and caregivers were instructed to keep at home isolation 14 days before the procedure, to report the presence of fever and to notify any COVID-19 contact or symptoms. Patients and donors were screened with a questionnaire prior to the start of conditioning and SARSCoV-2 PCR testing was added to the work-up after July 2020 .

The stem cell source was peripheral blood in all cases; the conditioning regimen was provided according to the diagnosis and has been previously described. [7] Briefly, for the allogeneic group (allo-HCT), this consisted on fludarabine (Flu) $25 \mathrm{mg} / \mathrm{m}^{2} /$ day and cyclophosphamide (Cy) $350 \mathrm{mg} / \mathrm{m}^{2} /$ day for 3 consecutive days, and melphalan (MEL) $70-100 \mathrm{mg} / \mathrm{m}^{2}$ or busulphan $4 \mathrm{mg} / \mathrm{kg}$ for 2 consecutive days, with or without 2 Gy of total body irradiation. Graft-versus-host prophylaxis included Cy $50 \mathrm{mg} / \mathrm{kg} /$ day on days +3 and +4 , mycophenolic acid $1 \mathrm{~g}$ /day from day +5 to day +35 , and oral cyclosporine (CsA) $5 \mathrm{mg} / \mathrm{kg} / \mathrm{day}$ from day +5 through day 180 , and then tapered to levels of $150-250 \mathrm{ng} / \mathrm{mL}$ for 30-60 days for the haploidentical group. For the HLA-identical recipients, oral CsA $5 \mathrm{mg} / \mathrm{kg} /$ day was administered on day -1 with intravenous methotrexate $5 \mathrm{mg} / \mathrm{m}^{2}$ on days $+1,+3$, and +5 , following the therapeutic scheme for CsA as with the haploidentical group. Autologous HCT (auto-HCT) recipients received Cy $50 \mathrm{mg} / \mathrm{kg}$ and etoposide $300 \mathrm{mg} / \mathrm{m}^{2}$ over 3 days. For multiple myeloma, Cy $600 \mathrm{mg} / \mathrm{m}^{2}$ and MEL $100 \mathrm{mg} / \mathrm{m}^{2}$ were administered for 2 consecutive days. After graft infusion, patients stayed at home and had daily follow-ups using telemedicine combined with scheduled visits to the hematology clinic every 3 days until engraftment was observed; symptomatic patients with bleeding, petechiae, or anemic syndrome were required to perform daily complete blood counts as needed for clinical stability. Mild to moderate complications were treated in an outpatient setting. Patients with bleeding, severe mucositis, persistent oral intolerance, diarrhea, uncontrolled neutropenic fever $\geq 38^{\circ} \mathrm{C}$, hemodynamic instability, 
Table 1 Operational changes during the COVID-19 pandemic for continuing hematopoietic cell transplantation (HCT) in the Hematology Department of the Dr. José E. González University Hospital of the Universidad Autónoma de Nuevo León, Monterrey, México.

\begin{tabular}{ll}
\hline Category & Operational changes \\
\hline Clinical Staff & Rotations were reduced to a minimum \\
- & All non-medical on-site activities were suspended (educational, laboratory or \\
voluntary work) & All staff wore personal protective equipment (facemask and shield) \\
- Entry points were limited to one to facilitate screening & All staff, patients or caregivers underwent active daily screening to identify \\
close contacts or presence of COVID-19 symptoms: \\
Triage
\end{tabular}

COVID-19 coronavirus disease 2019, HCT hematopoietic cell transplantation. severe cytokine release syndrome (CRS), and severe infection were hospitalized in isolation. If moderate to severe COVID19 developed, patients were admitted to the COVID-19 unit.

We performed a mean of 6.6 HCT per month in 2020 compared with 5.2 and 4.6 in 2019 and 2018, respectively (see Fig. 1a). Outpatient strategy was selected in $92 \%$ of our patients in 2020 in contrast to $70 \%$ in 2019. During the pandemic period $55.2 \%(n=32)$ HCTs were performed in patients referred from other institutions.

From March to December 2020, 58 patients underwent an outpatient HCT in our center, $33(57 \%)$ allo-HCT and 25 (43\%) auto-HCT. Allo-HCT were mostly from haploidentical donors $(n=25)$, with 8 from a HLA-identical donor. Most frequent diagnoses were acute lymphoblastic leukemia (ALL) $(n=10)$, multiple myeloma $(n=10)$, and lymphoma $(n=10)$. Thirty-five $(60.3 \%)$ patients completed the procedure without requiring hospitalization; 22 (62.8\%) auto-HCT and $13(37.1 \%)$ allo-HCT. From those requiring admission, the most common cause was febrile neutropenia (15/23 patients). Most common complications included infections $(n=15,48.3 \%)$ for allo-HCT and mucositis $(n=$ 9, 39.1\%) for auto-HCT. Acute graft-versus-host-disease (aGHVD) rates for haplo and HLA-identical-HCT were 48 and $25 \%$, respectively. CRS rate for the haploidentical group was $60 \%$ (15/25 patients), with all cases of mild severity (grade 1-2).
After transplantation, four patients $(6.8 \%)$ presented a positive SARS-CoV-2 PCR test and one (1.7\%) had clinical features suggestive of COVID-19 with a positive contact but a negative PCR test. Median time from HCT to COVID19 presentation was 25 days (4-138), one (20\%) received an auto-HCT, and four (80\%) an allo-HCT; three haploidentical and one HLA-identical. Two (40\%) patients from this group were pediatric, and three were adults $(60 \%)$. Three (60\%) patients with COVID-19 required hospitalization and none required mechanical ventilation nor died from the disease.

At last follow-up, four (6.8\%) patients were deceased at a median time of 115.3 (8-193) days after HCT. All these patients received an allo-HCT; two were haplo-HCT and two HLA-identical. All were hospitalized, and 1 (25\%) was admitted to the intensive care unit (ICU); causes of death were septic shock in $2(50 \%)$, and refractory/relapsed disease in two (50\%). Non-relapse mortality at day 100 was $7.9 \%$.

In the entire cohort, 100-day overall survival (OS) after HCT was 95.6\% (CI 95\%, 94.9-96.1), whereas patients receiving an allo-HCT presented a 100-days OS of $92.5 \%$ (CI 95\%, 91.4-93.5); no patient from the auto-HCT group died. When comparing OS of the pandemic cohort to a historical control of previous years treated at the same center, non-significantly lower OS was observed in the control group (86\% CI 95\%, 85.2-86.7, $p=0.219)$ (Fig. 1b). 


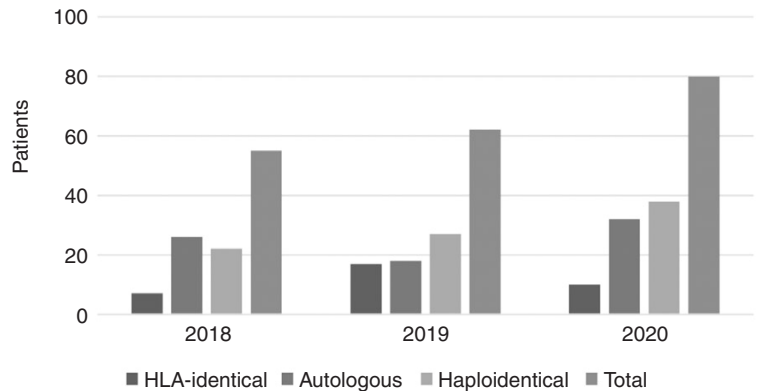

b

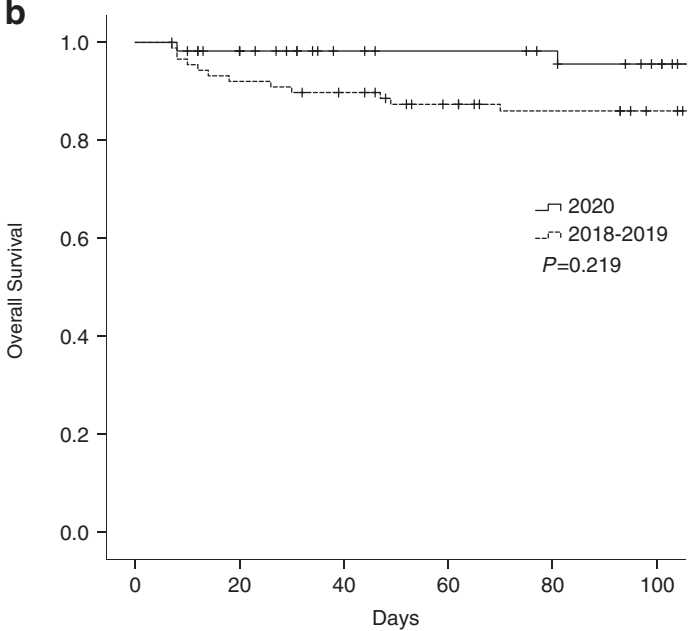

Fig. 1 Ambulatory HCT activity and survival rates at Dr. José Eleuterio González University Hospital in Monterrey, México. a Activity of the HCT program during the COVID-19 outbreak compared to the previous 2 years. There were 55 transplants in 2018, 62 in 2019, and 80 in 2020 (January-December). b Overall survival comparison between patients receiving an HCT during the pandemic period in 2020 and the pre-pandemic years 2018-2019.

We report the experience of transplant-related outcomes during the COVID-19 pandemic at a Hematology Center at a tertiary-care referral hospital. A previous descriptive study in Spain analyzed outcomes in 16 patients who underwent HCT between February 25 and June 15, 2020, reporting SARS-CoV-2 infection in $1(6.25 \%)$, suggesting that HCT is feasible. [6] In our study, which included 58 ambulatory HCTs, five patients (8.6\%) presented COVID-19 during the follow-up after HCT. In our survival analysis, compared to previous years, patients receiving HCT were not significantly affected by the pandemic in terms of mortality.

Moreover, comparing our results to a previous study conducted in our center between August 2013 and July 2017, other common complications such as infections showed similar proportions for allogeneic (48.3\% vs. 55\%) and autologous transplants $(27.3 \%$ vs. $28.9 \%)$ [7]. Also, the hospitalization rate was lower in the present report for both allogeneic $(60.6 \%$ vs. $64.9 \%)$ and autologous recipients (12\% vs. $28.9 \%)$. These findings support the idea of sustaining HCT conditions despite the SARS-CoV-2 outbreak, especially as other hospitals in our country that conducted HCT were forced to admit only COVID-19 patients [8], and patients that required HCT were referred to our center.

Previous studies have evaluated the impact of SARSCoV-2 infection in HCT recipients. Similarly to our study, one single-center analysis found a mortality rate of $28 \%$ within the first year after HCT [9]. However, long-term HCT recipients that get exposed to the virus are also at higher risk of complications than the general population. The CIBMTR recently published data of 318 HCT recipients that received a COVID-19 diagnosis at a median time of 17 and 23 months for allo-HCT and auto-HCT recipients after transplantation with an OS of $68 \%$ and $67 \% 30$ days after COVID-19 diagnosis, respectively. [10] These data highlight the need to implement strict hygiene preventive measures and aggressive treatment management in HCT recipients who develop COVID-19, even after the 100-days post-HCT have passed.

The outpatient method has played an essential role in the maintenance of our HCT program, as most of the transplantation costs are covered by the patient's own budget. To optimize resources and perform PCR tests when strictly necessary, donors were asked to isolate 2 weeks before mobilization, and PCR tests were performed only when the donor presented symptoms suggestive of COVID-19. This approach appears to be safe as there is no evidence that SARS-CoV-2 can be transmitted through blood products. [11]

Principal limitations in our report are its retrospective design and limited sample size. However, it describes a promising strategy for institutions without a conventional HCT unit.

This study demonstrates that a completely outpatient HCT is feasible and safe, while relieving stress on the healthcare system in the pandemic era, in which a rebound effect that overstresses transplantation programs has been anticipated. [12]

Acknowledgements We thank Sergio Lozano-Rodríguez, MD, for his critical review of this manuscript.

\section{Compliance with ethical standards}

Conflict of interest The authors declare no competing interest.

Publisher's note Springer Nature remains neutral with regard to jurisdictional claims in published maps and institutional affiliations.

\section{References}

1. The Lancet. COVID-19 in Latin America: a humanitarian crisis. Lancet 2020; 396:1463. 
2. Seber A. Transplantation during a worldwide crisis: lessons learned across the world. In: TCT transplantation \& cellular therapy meetings of ASTCT and CIBMTR digital experience. 2021. https://tct.confex.com/tct/2021/meetingapp.cgi/Paper/16601.

3. Ljungman P, Mikulska M, de la Camara R, Basak GW, Chabannon C, Corbacioglu S, et al. The challenge of COVID-19 and hematopoietic cell transplantation; EBMT recommendations for management of hematopoietic cell transplant recipients, their donors, and patients undergoing CAR T-cell therapy. Bone Marrow Transpl. 2020;55:2071-6.

4. Balduzzi A, Brivio E, Rovelli A, Rizzari C, Gasperini S, Melzi ML, et al. Lessons after the early management of the COVID-19 outbreak in a pediatric transplant and hemato-oncology center embedded within a COVID-19 dedicated hospital in Lombardia, Italy. Estote parati. Bone Marrow Transpl. 2020;55:1900-5.

5. Sahu KK, Cerny J. Managing patients with hematological malignancies during COVID-19 pandemic. Exp Rev Hematol. 2020;13:787-93.

6. Lázaro del Campo P, Ramírez López A, de la Cruz Benito B, de Paz Arias R, de Soto Álvarez T, Sánchez Vadillo I, et al. Hematopoietic cell transplantation during COVID-19 pandemic: experience from a tertiary hospital in Madrid. Exp Rev Hematol. 2021;14:1-5.

7. Jaime-Pérez JC, Salazar-Cavazos L, Aguilar-Calderón P, HerreraGarza JL, Gutiérrez-Aguirre CH, Gómez-Almaguer D. Assessing the efficacy of an ambulatory peripheral blood hematopoietic stem cell transplant program using reduced intensity conditioning in a low-middle-income country. Bone Marrow Transpl. 2019;54: $828-38$.

8. Rivera-Franco MM, Saldaña-Gonzalez ES, Leon-Rodriguez E. Changes within the care of hematopoietic cell transplantation patients after the shift of a Mexican institution to a COVID-19only center. Hematol Transfus Cell Ther. 2020;42:313-5.

9. Kanellopoulos A, Ahmed MZ, Kishore B, Lovell R, Horgan C, Paneesha S, et al. COVID-19 in bone marrow transplant recipients: reflecting on a single centre experience. Br J Haematol. 2020;190:e67-e70.

10. Sharma A, Bhatt NS, St Martin A, Abid MB, Bloomquist J, Chemaly RF, et al. Clinical characteristics and outcomes of COVID-19 in haematopoietic stem-cell transplantation recipients: an observational cohort study. Lancet Haematol. 2021;3026: $1-9$.

11. Leclerc M, Fourati S, Menouche D, Challine D, Maury S. Allogeneic haematopoietic stem cell transplantation from SARS-CoV2 positive donors. Lancet Haematol. 2021;2:9-10.

12. Willan J, King AJ, Djebbari F, Turner GDH, Royston DJ, Pavord $\mathrm{S}$, et al. Assessing the impact of lockdown: fresh challenges for the care of haematology patients in the COVID-19 pandemic. Br J Haematol. 2020;189:e224-e227. 